\title{
¿QUÉ SABEMOS SOBRE EL DUELO Y EL FINAL DE LA VIDA LAS ENFERMERAS? A PROPÓSITO DE UN ESTUDIO MEDIANTE GRUPO FOCAL EN SANTA CRUZ DE TENERIFE
}

\author{
Cristo Manuel Marrero González* \\ Alfonso Miguel García Hernández** \\ Universidad de La Laguna
}

\section{RESUMEN}

Introducción y propósito de la investigación: los autores, miembros del grupo de cuidados al final de la vida de la Universidad de La Laguna, buscan identificar áreas de mejora con respecto al cuidado al final de la vida, las pérdidas y el duelo en el contexto profesional, desde la premisa de que es escasa e insuficiente la formación y preparación en torno al tema que nos trae. Metodología: se trata de una investigación fenomenológica hermenéutica mediante grupo focal de quince enfermeras del centro de salud Ofra Delicias-Miramar, en Santa Cruz de Tenerife (España), realizada en noviembre de 2019. Resultados: se identifican cuatro categorías principales al profundizar en ¿qué es el duelo?: el espacio físico del duelo y la muerte; el significado y contexto; el duelo por niños fallecidos; y propuestas de mejora. Conclusiones: las enfermeras refieren no sentirse suficientemente preparadas y formadas en lo que respecta al duelo y el proceso de morir. La formación es escasa y cabe plantearse y reflexionar sobre cómo las enfermeras realizan diagnósticos, establecen objetivos e intervenciones a personas en el final de la vida o en el proceso de duelo.

Palabras Clave: enfermeras, formación, pérdida y duelo.

\section{WHAT DO NURSES KNOW ABOUT BEREAVEMENT AND END OF LIFE CARE? INVESTIGATION TROUGH FOCUS GROUP IN SANTA CRUZ DE TENERIFE}

\begin{abstract}
Introduction and purpose of the research: the authors, members of the End-of-Life Care group at the University of La Laguna (CUFINVIDA), seek to identify areas of improvement concerning end-of-life care, loss and grief in the professional context, from the premise that there is not enough training around this topic. Methodology: phenomenological hermeneutic research through a focus group of fifteen nurses from Ofra Delicias-Miramar primary healthcare centre in Santa Cruz de Tenerife (Spain), carried out in November 2019. Results: Four main categories are identified when delving into what mourning is: the physical space of mourning and death; meaning and context; mourning for deceased children and proposals for improvement. Conclusions: Nurses report not feeling prepared regarding grief and the process of dying. Training is scarce, and it is necessary to consider and reflect on how nurses carry out diagnoses and establish objectives and interventions for people at the end of life or in the mourning process.
\end{abstract}

KEYWORDs: nurses, training, loss and grief.

DOI: https://doi.org/10.25145/j.cuidar.2020.00.05

Revista CuIDAR, 0; junio 2020, pp. 61-69; ISSN: 2660-4426 


\section{ANTECEDENTES. JUSTIFICACIÓN DE LA PRESENTE INVESTIGACIÓN}

Los autores del presente estudio son miembros del grupo de investigación de la Universidad de La Laguna Cuidados al Final de la Vida (End of Life Care) y por tanto la misión de nuestro grupo, como menciona García Hernández (2017), es «[...] ofrecer instrumentos para estudiar la diversidad de los cuidados y planteamientos en torno al final de la vida analizando los factores que facilitan y/o propician un morir más "humano" en el contexto de unos procesos claramente culturizados".

Y el artículo de revisión «Vivencias de las enfermeras ante la muerte. Una revisión» (Marrero González y García Hernández, 2019) aborda y reflexiona en el dinámico y complejo proceso de morir y el duelo, temas escasamente abordados en nuestra profesión enfermera. Consultando los trabajos para elaborar dicho ensayo, nos percatamos de que era necesario realizar una investigación en nuestro entorno social, laboral y profesional actual en la isla de Tenerife (España) que sirviera de punto de partida a la visión y/o percepción que tienen las enfermeras sobre el duelo, ante la escasez de trabajos en este sentido. Para ello se eligió como lugar de estudio un centro sanitario. En concreto, el centro de atención primaria de salud Ofra Delicias-Miramar, en la ciudad de Santa Cruz de Tenerife.

Se ha requerido la participación del doctor Alfonso Miguel García Hernández, por su experiencia vital, profesional, investigadora, docente y académica en el campo del duelo y el cuidado al final de la vida. Su aportación a este trabajo de investigación ha sido de suma importancia para orientar el modelo de investigación y para el asesoramiento en la categorización de los resultados.

\section{INTRODUCCIÓN}

El presente trabajo busca profundizar en los conocimientos que poseen las enfermeras acerca del duelo, su proceso y la vivencia de la muerte. Por ello nos aproximamos a un grupo de enfermeras que trabaja en el centro de salud Ofra Delicias-Miramar (Santa Cruz de Tenerife, España) y mediante grupo focal exploramos estas cuestiones de modo que nos permitan deducir qué conocimiento tienen al respecto las enfermeras, cuál es su formación, extrapolando las mismas a las enfermeras de Tenerife en este sentido; cómo el contexto y la experiencia vivida las lleva a

* Enfermero en el Servicio Canario de la Salud. Área de salud de Tenerife (España). Doctor por la Universidad de La Laguna. Miembro del grupo de investigación de Cuidados al Final de la Vida (End of Life Care) de la Universidad de La Laguna. Correo electrónico: cmmarrerogonzalez@ gmail.com.

** Licenciado en Enfermería. Doctor en Antropología Social y Cultural por la Universidad de La Laguna. Profesor titular de la Universidad de La Laguna (España). Coordinador del grupo de investigación de Cuidados al Final de la Vida (End of Life Care) de la Universidad de La Laguna. 
explicar sus vivencias en lo que se refiere a la muerte y el duelo de sus pacientes o personales. Reflexionar en este sentido sobre la muerte y el duelo a lo largo de las distintas edades de la vida desde la percepción de las quince enfermeras que trabajan en dicho centro de salud nos va a permitir establecer objetivos y áreas de mejora en lo que respecta a la atención al final de la vida, las pérdidas y el duelo en el contexto profesional.

El estudio que realizamos comporta un entendimiento fenomenológico hermenéutico con análisis del discurso y en el mismo participan un grupo de quince enfermeras pertenecientes al centro de salud Ofra Delicias-Miramar, en Santa Cruz de Tenerife (España). Los participantes son 14 mujeres y un hombre, de edades comprendidas entre 26 y 56 años.

Tras explicar a los quince enfermeros en qué consiste la reunión del grupo focal y la investigación planteada, se hace entrega del documento de consentimiento informado, que firman la totalidad de los miembros, que recoge la garantía de confidencialidad y anonimato de los participantes.

De acuerdo con los objetivos propuestos, los temas planteados en el grupo focal son los siguientes: ¿qué saben acerca del duelo?, ¿cómo viven el hecho de que pueda fallecer un paciente?, ¿siempre se han sentido preparadas para afrontar un final de vida?, ¿qué les supone perder a un paciente joven?, ¿'y uno anciano?, ¿creen que han sido suficientemente formadas sobre cómo afrontar el proceso de muerte y duelo? Y, por último, desde el punto de vista de ustedes, ¿qué mejoras propondrían?

Tras la recogida de datos grabados en audio-formato mp3 se procede a la transcripción del grupo focal en formato Word. Se realiza selección, segmentación, codificación y categorización de los resultados obtenidos.

El grupo focal se realizó el 13 de noviembre de 2019. Tras la recogida de los datos en dicho día, se comienza con la transcripción de la información para proceder a la categorización de los datos. La categorización es realizada en febrero de 2020.

\section{RESULTADOS}

Trabajar con compañeros de profesión siempre es revelador y enriquecedor, porque, además de compartir, nos permite profundizar intelectualmente en los conceptos de muerte y duelo, además de conocer la ignorancia que el mismo genera a la vez, los itinerarios personales y profesionales del mismo, sus pensamientos, acciones y sentimientos que en gran parte de las ocasiones van de la mano.

Las categorías y subcategorías que refieren son:

\begin{tabular}{ll}
\hline \multicolumn{1}{c}{ Categorías } & \multicolumn{1}{c}{ Subcategorías } \\
\hline 1. ¿Qué es el duelo? & $\begin{array}{l}\text { 1.1. Las vivencias y reflexiones personales sobre el morir } \\
\text { y el duelo. } \\
\text { 1.2. Casos cercanos. }\end{array}$ \\
\hline $\begin{array}{l}\text { 2. El espacio físico del duelo y la muerte: } \\
\text { significado y contexto. }\end{array}$ & 1.3. La problemática de morir en el domicilio. \\
\hline
\end{tabular}



tarlas.

Realizada la descripción y categorización de las mismas, pasamos a comen-

Categoría 1. ¿Qué es el Duelo?

En la presente categoría, las participantes describen de una forma reflexiva y personal qué es para ellas el duelo. Las descripciones que realizan acerca del duelo se basan en lo aprendido, principalmente desde sus experiencias profesionales y vitales. Plantean poca formación profesional, aunque tratan de dar una definición conceptual del duelo. Existe una percepción de que el duelo en el contexto actual se vive de forma fría.

El duelo es un proceso natural (enfermera 4).

El duelo es un proceso que consta de varias fases que cada uno lo vive a su manera condicionado por sus vivencias, su cultura y todo lo que le rodea. Últimamente se tiende a aislar a la gente, se les dejan solos, como que lo tienes que resolver tú solo. Creo que hace falta que la gente se acerque, con más cariño, con más escucha. Se normaliza mucho la muerte (enfermera 1).

Es un proceso difícil de resolver. Tenemos unos conceptos de seis meses o un año natural. Es un proceso muy lento. Aunque sea algo natural crea mucho dolor y mucho vacío [...] (enfermera 3).

\subsection{Las vivencias y reflexiones personales sobre el morir y el duelo}

Acerca del concepto de duelo y del significado de muerte, las enfermeras reflexionan sobre la longevidad de vida o la dificultad por aceptar que la muerte llega a cualquier persona y a cualquier ser querido. La muerte es algo natural y no debería haber tanto encarnizamiento terapéutico en muchos casos como en personas muy añosas. La mayor esperanza de vida, los adelantos terapéuticos han hecho que se cueste más aceptar que un ser querido tiene que fallecer, porque es un hecho natural e inevitable. Por lo que manifiestan las enfermeras, en nuestro contexto es más difícil aceptar la muerte y aún más si se trata de alguien relativamente joven.

La muerte es algo inevitable (enfermera 9).

Estamos acostumbrados a que la gente no se tiene que morir. No aceptamos la muerte de los que se van a morir [...] hay dos realidades en que se nos pone un tiempo o un precio al duelo, pero también está la realidad de que la sociedad nos ha maleducado en el sentido de que parece que ahora no hay que morirse y hay que luchar contra viento y marea (enfermera 7). 
Tengo muchos pacientes de 90 y tantos años y la familia quiere luchar y luchar para mantenerlo en vida. No es la enfermedad, es la naturaleza y les cuesta tanto aceptarlo y quieren ingresarlo (enfermera 4).

Lo que no es normal es que haya unos duelos larguísimos de tu abuela de 100 años. Es la no aceptación de que la vida se acaba [...] morirse no es un fracaso (enfermera 7). Vi un duelo y entierro en Bali de un niño de ocho años y se veía a niños vestidos de blanco cantando porque creen que van a otra vida. Ellos no lo sufren como lo sufrimos nosotros porque culturalmente es diferente. Pienso que como lo hayamos vivido y aprendido, lo entendemos. A mí me chocó muchísimo ver aquello (enfermera 8).

Hay más dolor por una persona joven. Más difícil de aceptar (enfermera 8).

\subsection{Casos cercanos}

En esta subcategoría, las enfermeras expresan reflexiones sobre cómo es el duelo, el final de vida y cómo se viven estos procesos desde casos personales con seres queridos o familiares. No llega a formar un corpus de conocimiento, aunque sí una orientación vital.

Lo peor que he vivido era ver la agonía en los últimos momentos de la vida y no poder hacer nada. Ese momento es terrible (enfermera 3).

Yo viviría la muerte de mi madre de manera más natural que la muerte de mi hijo. Veo más natural pasar el dolor como hija que como madre (enfermera 10).

Yo perdí a mi padre hace cinco ańos y a mi abuela este ańo. Considero que los dos duelos han sido diferentes. Con el de mi padre pude identificar las distintas etapas por las que iba pasando, o sea, la ira, la negación... Y ahora estoy más aliviada. La de mi abuela fue más natural. Todo depende de la persona que sea, el vínculo que tengas con ella (enfermera 8).

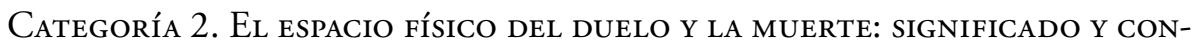 TEXTO}

Este tema o categoría surge a raíz de la reflexión del grupo focal sobre los ritos actuales en torno al duelo y el morir en el contexto de la sociedad moderna. Al considerar que el espacio físico relativo a las muertes, alejado del espacio íntimo del hogar y su traslado a tanatorios o morgues, lo ha transformado en espacios públicos más fríos en los que se echa en falta el poder decidir cómo y dónde velar al difunto, unificando y estereotipando el proceso.

En este país se ha pasado en 50 años de los velatorios que era toda una realidad, un ritual de luto que tenía que alargarse al menos un año. No se ha aceptado que es un hecho natural, sino que debe acortarse con incluso medicación para ser tratado. Han cambiado los rituales de normas sociales (enfermero 5).

[...] Antes los velatorios eran en las casas e iba la gente, los vecinos... Ahora se ha aislado. Todo se hace en sitios muy asépticos, muy concretos como los tanatorios. 
Está apartado de la vida diaria. Todo contribuye a dejarlo fuera de contexto (enfermera 2).

Yo pensaba que lo más natural era pasar el duelo en casa, pero no me lo permitieron. Tuvimos que hacerlo en el tanatorio [...] (enfermera 3).

\subsection{La problemática de morir en el domicilio}

A colación del cambio de lugar o espacio donde velar a los difuntos, surge también el debate o reflexión sobre el hecho de poder morir en el domicilio y no en una institución sanitaria. Desde las dificultades que ello comporta para poder decidir por parte de pacientes y familiares dónde desean que sea su lugar de fin de vida. Además de que existe miedo o temor por no saber cómo actuar ante un proceso de muerte agónico en casa.

[...] Los familiares tienen miedo de que su ser querido muera en casa. Creo que debemos transmitirles a los familiares que se trata de un proceso natural. Es un proceso que se debe vivir en casa (enfermera 3 ).

Con los cuidados paliativos actuales no te ves en tu casa protegido. Los familiares siempre se ven en la obligación de tirar hacia urgencias. La gente se niega a acudir a urgencias con un familiar terminal. Los cuidados paliativos a domicilio faltan (enfermera 6).

Hay poco apoyo para tomar la decisión de morir en casa. Es una decisión un poco falsa (enfermera 7).

A veces teniendo los apoyos tenemos miedo a que nuestro familiar muera en casa (enfermera 6).

\section{Categoría 3. Duelo por niños Fallecidos}

La pérdida, y en particular la vivida tras la muerte de un hijo, se plantea como diferente, dolorosa y varía en función del proceso de muerte de los hijos fallecidos. Un diagnóstico repentino e impredecible se comporta de modo diferente que una enfermedad terminal, y se vive como un proceso más doloroso y terrible como atestigua una enfermera. La construcción de los significados dado al proceso y la participación y vivencia del mismo hacen que se afronte mejor la situación por parte de padres y madres.

El trato con los padres es lo importante. En estos últimos siete ańos se me han ido algunos nińos. Uno con parálisis cerebral y un niño con un tumor cerebral. Este último caso fue aplastante porque duró un mes. El caso de la parálisis cerebral fue más tratable. Porque es a largo plazo. Tienes un tiempo para hablar con los padres. Los padres son más conscientes ya que desde que nacen saben que su esperanza de vida es muy corta y están rodeados de muchos profesionales que les ayudan. En el caso del niño con el tumor cerebral sus padres estaban muy desamparados. No hay apoyo para esos padres (enfermera 11). 
Las propuestas de mejora que se establecen se orientan principalmente a una mejora de los cuidados paliativos y de la atención en el cuidado al final de la vida por parte de la sanidad pública y en segundo lugar porque haya más y mejor formación en duelo y final de vida para las enfermeras.

\subsection{Mejora de los cuidados paliativos y la atención domiciliaria al final de la vida}

En esta subcategoría las enfermeras hacen referencia a que la cobertura del servicio de cuidados paliativos es limitada, al igual que la atención domiciliaria paliativa. Hay escasos recursos humanos y un vacío de comunicación entre atención primaria y atención especializada. El cuidado al final de la vida deseado necesita de ayuda al familiar además de preparación al mismo en el proceso de morir.

Enfermería tiene que preparar a la familia. Eso es un proceso largo. Es un proceso de formación al familiar de lo que va a ocurrir. Hay que contar con todo el equipo (enfermera 3).

Necesitamos más comunicación con el servicio de paliativos. Se necesitan más recursos humanos (enfermera 6).

Lo importante es potenciar los cuidados paliativos y el morir sin dolor (enfermera 8).

\subsection{Mayor y mejor formación}

El punto de partida por las distintas enfermeras es que existe escasa formación sobre el final de vida y sobre el duelo. Reconocen en este sentido sus limitaciones y escasos conocimientos, y hacen énfasis en que todo lo que se ha podido aprender no ha sido en base a formación, sino por experiencias vitales en lo personal y en lo profesional como se ha venido describiendo con anterioridad.

Necesitamos más formación. Yo no tengo ni idea (enfermera 4).

No se nos ha formado lo suficiente. Es por lo que has vivido, lo que has visto o leído un poco (enfermera 6).

Para la práctica no se nos ha formado (enfermera 2).

Yo lo he aprendido de forma natural y así se lo he transmitido a mis hijas. Cada una tiene su forma de aceptarlo (enfermera 3).

\section{CONCLUSIONES. REFLEXIONES FINALES}

Las enfermeras refieren que no se encuentran lo suficientemente preparadas y formadas para los procesos de duelo y final de vida. Una afirmación que no se basa solo en lo que diferentes profesionales ya han avanzado al respecto, sino también porque es reconocido por parte de las enfermeras que han participado en 
el presente estudio. La formación es muy escasa por no decir prácticamente nula, lo cual nos lleva a reflexionar y preguntarnos por cómo se realizan diagnósticos, se establecen objetivos e intervenciones enfermeras a personas que viven el final de la vida o a lo largo de un proceso de duelo.

\section{AGRADECIMIENTOS}

Los autores agradecen encarecidamente la buena predisposición de la dirección del centro de salud de atención primaria Ofra Delicias-Miramar para realizar el grupo focal y en particular agradecemos a las enfermeras su participación en este estudio. Sirva también para adelantar que dichas profesionales recibirán formación por parte del Dr. Alfonso Miguel García Hernández en diferentes sesiones clínicas en torno al citado tema.

\section{CONFLICTO DE INTERESES}

Los autores declaran que no ha habido conflicto de intereses durante el proceso de investigación. 


\section{BIBLIOGRAFÍA}

García Hernández, A.M. (2017). El Grupo de Investigación de la Universidad de La Laguna Cuidados al Final de la Vida. Ene. Revista de Enfermería, 11(2), 0-0.

Marrero González, C. y García Hernández, A. (2019). Vivencias de las Enfermeras ante la Muerte. Una Revisión. Revista ENE de Enfermería, 13(2), 0-0. 
